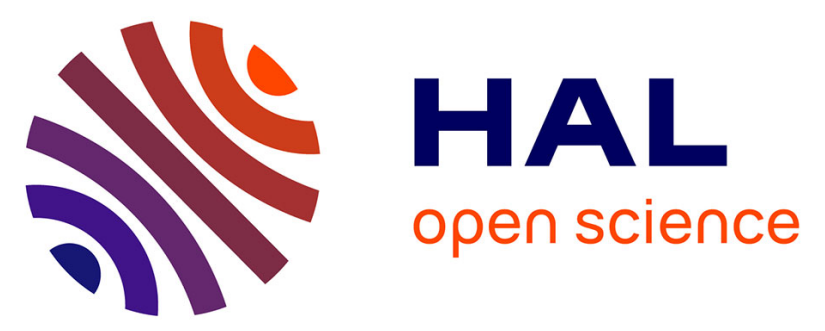

\title{
Stimulated Raman scattering for variable gain amplification of small optically carried microwave signals
}

Kafing Keïta, Robert Frey, Philippe Delaye, Daniel Dolfi, Jean-Pierre Huignard, Gérald Roosen

\section{- To cite this version:}

Kafing Keïta, Robert Frey, Philippe Delaye, Daniel Dolfi, Jean-Pierre Huignard, et al.. Stimulated Raman scattering for variable gain amplification of small optically carried microwave signals. Optics Communications, 2006, 263, pp.300-303. 10.1016/j.optcom.2006.01.027 . hal-00673997v2

\section{HAL Id: hal-00673997 https://hal-iogs.archives-ouvertes.fr/hal-00673997v2}

Submitted on 23 Mar 2012

HAL is a multi-disciplinary open access archive for the deposit and dissemination of scientific research documents, whether they are published or not. The documents may come from teaching and research institutions in France or abroad, or from public or private research centers.
L'archive ouverte pluridisciplinaire HAL, est destinée au dépôt et à la diffusion de documents scientifiques de niveau recherche, publiés ou non, émanant des établissements d'enseignement et de recherche français ou étrangers, des laboratoires publics ou privés. 


\title{
Stimulated Raman scattering for variable gain amplification of small optically carried microwave signals
}

\author{
Kafing Keita ${ }^{(1,2)}$, Robert Frey ${ }^{(1)}$, Philippe Delaye ${ }^{(1)}$, Daniel Dolfi ${ }^{(2)}$, Jean- \\ Pierre Huignard ${ }^{(2)}$, Gérald Roosen ${ }^{(1)}$ \\ (1) Laboratoire Charles Fabry de l'Institut d'Optique, Centre National de la Recherche \\ Scientifique, Université Paris-Sud, Centre Scientifique Paris-Sud, Bât. 503, F-91403 Orsay \\ cedex, France.
}

(2) Thales Research \& Technology, RD 128, F-91767 Palaiseau cedex, France

\begin{abstract}
Stimulated Raman amplification of optically carried microwave signals in optical fibers is experimentally investigated. In the backward configuration which is shown to be less noisy than the forward one, net amplification factors larger than $20 \mathrm{~dB}$ are demonstrated in the low signal regime with better relative intensity noise (RIN) values than using erbiumdoped fiber amplifiers. Due to the RIN flatness with respect to the gain of the stimulated Raman amplifier high quality variable gain amplification of optically carried microwave signals could be implemented using optimized Raman amplifiers
\end{abstract}

\section{Introduction:}

Distribution and processing of optically carried microwave signals are among the basic functions for future active radar antennas [1]. However, due to the high linearity performances required for applications, the radio-frequency (RF) signal is encoded by means of integrated modulators on an optical carrier at low optical power with very small modulation depths [2]. Moreover, carrier depletion (for instance using stimulated Brillouin scattering $[3,4]$ ) further reduces the optical power, making optical amplification necessary. This amplification, generally operated using Erbium-doped-fiber-amplification (EDFA) [5,6], 
is accompanied by amplified spontaneous emission (ASE) noise. This contribution can be particularly large for the strong amplification of the small signals encountered in the field of optically carried microwave signals since, in that case, the amplifier is not saturated by the signal intensity and the noise level will be large.

Here we show that stimulated Raman amplification (SRA) in silica fiber can be used for low noise amplification of such small optically carried microwave signals. SRA in silica fibers has been widely studied [7] and successfully applied [8] in optical telecommunications essentially due its extremely large bandwidth $(2.4 \mathrm{THz})$ which makes this amplification process very appropriate to dense wavelength-division-multiplexing (DWDM). However, even if several results obtained in these previous studies may be applied to microwave fiber optic links, the problem is fundamentally different essentially because of the nature of the optical signals to be amplified in RF photonics. Indeed, DWDM communications deal with digital short duration signals carried by discrete wavelengths in which the main aim is to discriminate two discrete intensity level representing " 0 " and " 1 " bits, while microwave optoelectronic links use optically carried analogical high frequency RF signals for which we want a minimum degradation of the frequency purity of the signal, with a large rejection of noise around the chosen RF. This means that the large bandwidth of SRA in silica fibers is not useful in this last case. However, due to its high gain SRA in fibers can be very attractive for the purpose of low noise small signal amplification of optically carried RF signals and to our knowledge little attention has been paid on this capability for microwave photonics applications. In particular, a significant advantage is that in SRA the small signal amplification gain and the total spontaneous noise power are both proportional to the pump power [9]. This peculiarity of SRA can ensure a constant output relative intensity noise (RIN) value with respect to gain variations, a property which cannot be achieved using EDFA [10] thus leading to the development of high performance variable gain amplifiers. In the following we experimentally and theoretically demonstrate this new advantage of SRA over Erbium doped fibers amplifiers for microwave fiber optic links.

Experiment:

The Raman amplifiers studied in our analysis consisted of conventional singlemode fibers of different lengths pumped at a wavelength of $1480 \mathrm{~nm}$ by a CW fiber Raman laser ( $2 \mathrm{~W}$ power and $1 \mathrm{~nm}$ linewidth). In the $\mathrm{CW}$ regime two configurations with co- and counterpropagating signal and pump beams are possible (Fig. 1). Nevertheless, as well know, the copropagating configuration presents several constraints related to the presence of Modulation Instabilities [11] and to the easier transfer of the pump noise towards the signal beam [12], 
that would give rise to troubles for the realization of a low noise amplification link. This additional noise was effectively observed (Fig. 2), that's why our experiments focus on the counter-propagating geometry (Fig. 1).

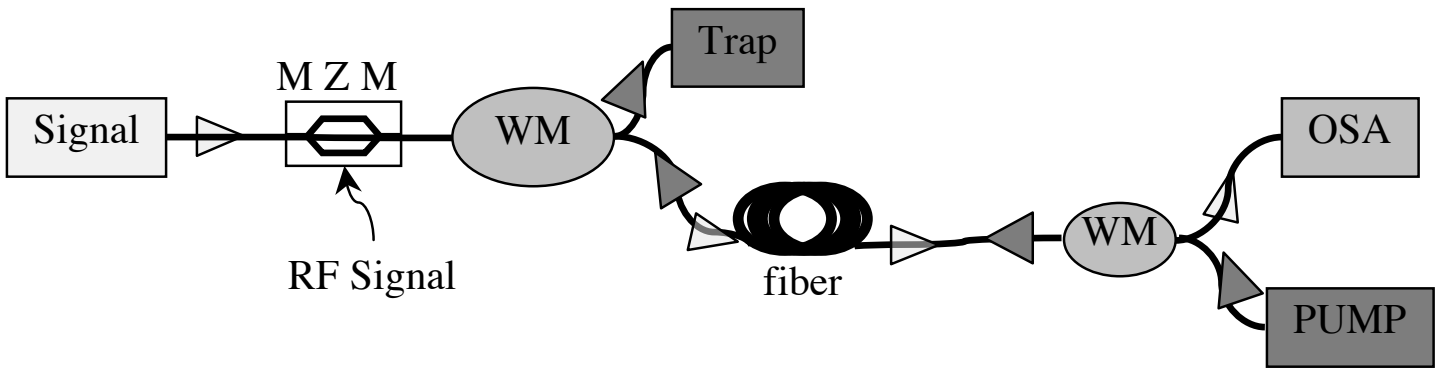

Figure 1 : Experimental setup for counter-propagating SRA. MZM : Mach-Zehnder modulator. WM : wavelength multiplexer. OSA : optical spectrum analyser. In the co-propagating scheme, we only had to exchange the position of the pump laser and trap.

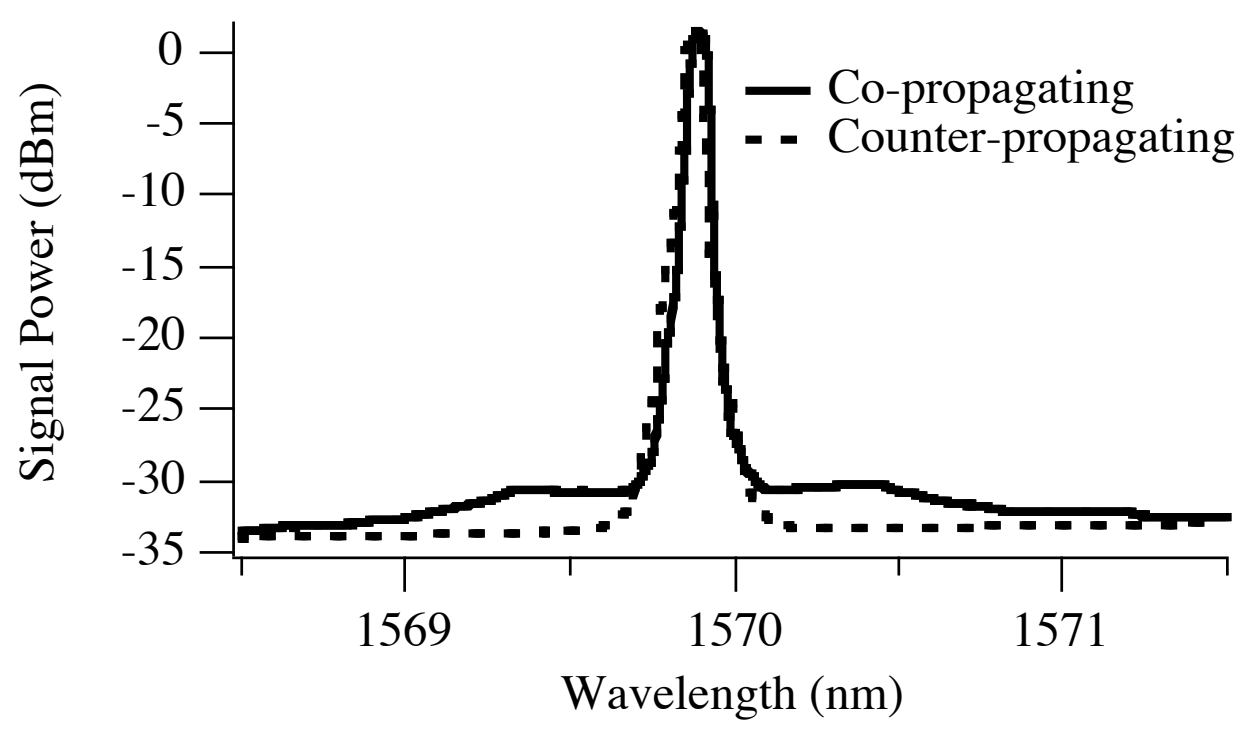

Figure 2 : Signal power spectrum for co- and counter-propagating SRA. The fiber length is $22.5 \mathrm{~km}$, and the pump power is $1.7 \mathrm{~W}$ in both cases, leading to a net gain of $25 \mathrm{~dB}$.

The signal beam was delivered by a low noise tunable semiconductor laser $(0.01 \mathrm{~mW}$ power, $1570 \mathrm{~nm}$ wavelength and $73 \mathrm{~dB}$ SNR optically measured in a wavelength bandwidth of $0.04 \mathrm{~nm}$ ) coupled to an integrated Mach-Zehnder interferometer modulated at a frequency of $20 \mathrm{GHz}$. The resulting modulated beam was injected in the Raman fiber amplifier using a wavelength multiplexer that also extracts the residual pump beam which is stopped in a trap. At the fiber end, the amplified signal beam is extracted by a second wavelength multiplexer 
that also injects the counter-propagating pump beam in the fiber. The signal beam was then sent in an optical spectrum analyzer. For comparison, measurement with a commercial EDFA (Keopsys, KPS-BT2-C27-PB-FA) were performed. Due to its architecture the EDFA replaces the whole system between wavelenth multiplexers (pump included too), and the measurement was made without any additional fiber.

\section{Results:}

In the different experiments we measure the net amplification factor (defined as the ratio of input and output signal powers), as well as the noise level. Figure 3 shows spectra of the injected and amplified signal beams modulated at a frequency of $20 \mathrm{GHz}$ (dotted and continuous lines respectively) and the ASE obtained with no signal beam (dashed line) for a $22.5 \mathrm{~km}$ long fiber and a pump power of $1.6 \mathrm{~W}$. The net amplification factor was $24 \mathrm{~dB}$ and no degradation of the signal beam was observed. Although a more quantitative analysis has to prove the spectral uniformity of the amplification factor which could be affected by four wave mixing processes, this first result is a strong indication of the usefulness of SRA to amplify optically carried microwave signals. Moreover, as expected for an unsaturated amplifier, the noise level was not influenced by the presence of the signal beam (compare continuous and dashed lines out of the laser line regions in fig. 3).

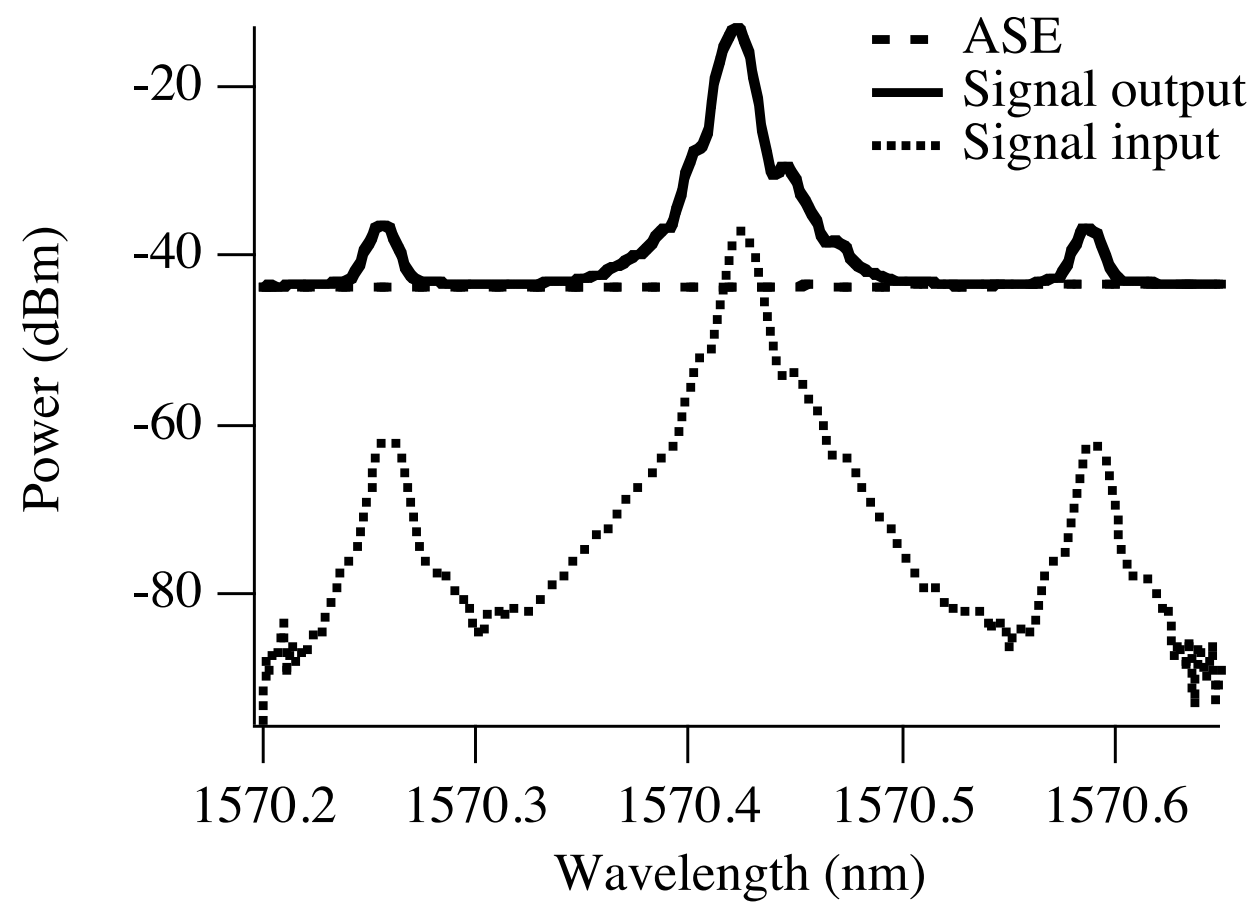

Figure 3 : Input and output signal and ASE spectra. 
In order to compare the respective influence of ASE in EDFA and SRA experiments were also performed by replacing the SRA by an EDFA. This comparison is illustrated in figure 4 showing the mean relative intensity noise $\mathrm{RIN}=\mathrm{SNR}^{-2} / \Delta \mathrm{F}$ plotted as a function of the net amplifier gain $\mathrm{G}=\mathrm{P}_{\text {Sout }} / \mathrm{P}_{\text {Sin }}\left(\mathrm{SNR}=\mathrm{P}_{\text {Sout }} / \mathrm{P}_{\mathrm{ASE}}\right.$ is the optical signal to noise ratio, with $P_{\text {Sin }}=-20 \mathrm{dBm}$ and $P_{\text {Sout }}$ the input and output signal powers and $P_{A S E}$ the noise power resulting from the amplification of photons emitted through spontaneous Raman generation, and $\Delta \mathrm{F}=8.56 \mathrm{GHz}$ is the bandwidth of the optical analyzer). For SRA, due to fiber losses, shorter was the fiber lower was the RIN value with minimum values around $-168 \mathrm{~dB} / \mathrm{Hz}$ for a fiber length of $6.5 \mathrm{~km}$. In this case, however, the maximum gain achievable in our experiment was only $12 \mathrm{~dB}$. Note, however, that with the same fiber length, higher Raman gains could be possible using the peak gain of the Raman transition, fibers with higher Raman nonlinearities such as Ge-doped fibers or higher pump powers. The measured values are also in very good agreement with those calculated using equations given in Ref.13, as it can be seen in fig. 4 showing fits obtained for $\mathrm{L}=6.5$ and $21 \mathrm{~km}$ (bolded lines). Let us also note the flatness of the RIN with respect to the net gain of the amplifier whatever the fiber length is. This RIN flatness which is obviously connected to the independence of the Raman-gain-tospontaneous-emission ratio from the pump power [9] is very interesting for high quality variable gain amplifiers of optically carried microwave signals. 


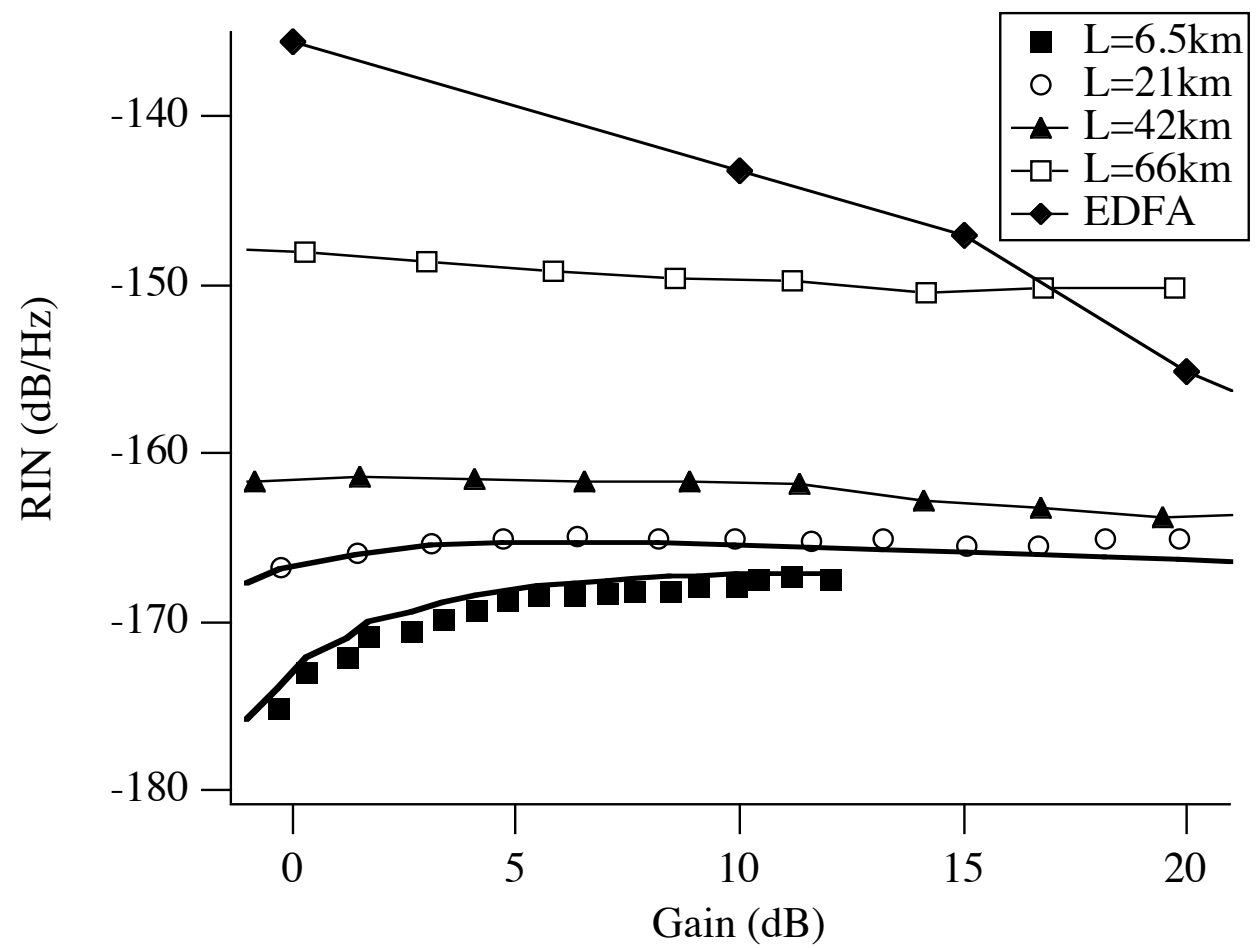

Figure 4 : Compared RIN for SRA and EDFA as a function of amplifier net gain for a $-20 \mathrm{dBm}$ input signal. Light solid lines are guides for the eyes while bolded solid lines correspond to fits obtained using equations giving the amplitude of the amplified spontaneous emission responsible for the noise given in Ref. 13

For comparison with existing amplification systems used in microwave fiber optic links [5,6], we make a measurement with an EDFA system. The RIN of the EDFA exhibits much higher values (see closed diamonds in fig. 4) and we clearly see that the RIN improvement found when using SRA was between 30 and $10 \mathrm{~dB} / \mathrm{Hz}$ depending on the gain value. This is, in fact, directly connected to the decrease of the EDFA RIN value with increasing net gains (see fig. 4) which appears due to the decrease of the amplifier population inversion factor [10].

The present study just considers SRA and EDFA independently of the system in which they will be inserted. The final choice may depend on the exact architecture of the microwave fiber optic link and essentially the length of the transmission fiber. In short links, the transmission fiber brings only negligible losses but can not be used to perform SRA, that will then use its own specific fiber for amplification (this case corresponds to the one described in Fig. 4). With long transmission fibers, the fiber will be directly used to implement the SRA, without additional fiber whereas the losses induced by the transmission fiber will bring additional parameters in the evaluation of the EDFA performances. In this last case the 
influence of the transmission line will depend on the position of the EDFA in the system and will need a careful evaluation. If positioned after the transmission fiber, the input signal power will be small and the EDFA will be used far from saturation with a higher noise figure. On the other hand if used as a booster before the launching of the signal in the transmission fiber, the EDFA may be operated closer to the saturated regime (the reduced net gain due to losses of the fiber would then correspond to a shift of the EDFA curve to the left in Fig. 4) but the high power signal may then induce nonlinear effects in the transmission fiber (such as Stimulated Brillouin Scattering) which will decrease the transmission performances.

The low RIN value measured for SRA and the flatness of this RIN with the gain value demonstrate the superiority of Raman amplification for small signal amplification of optically carried microwave signals with variable gain. Evidently, this optical measurement which is averaged on a large frequency bandwidth does not provide as much information as electrical measurements can and these last should be done for definite conclusions. However, as SRA does not suffer from relaxation oscillations our measurements provide a good first indication.

\section{Conclusion:}

Amplification of optically carried microwave signals have been demonstrated using stimulated Raman amplification in conventional telecom optical fibers. For backward SRA which is known to be less noisy than forward SRA, net amplification factors larger than $20 \mathrm{~dB}$ have been obtained in the small signal regime together with an improvement of the RIN value around 10 to $30 \mathrm{~dB} / \mathrm{Hz}$ when compared to EDFA. Even better results are expected using an optimized SRA. Preliminary results presented in this paper tend to prove that SRA can provide an attractive mechanism of low noise and variable gain amplification for the transmission and the processing of optically carried RF signals.

\section{References}

[1] H. Zmuda, E. N. Toughlian, Photonic Aspects of modern radars, Artech House, Boston, 1994.

[2] F. Heismann, S. K. Korothy, and J. J. Veselka in Optical fiber telecommunications, vol. III B, I. P. Kaminow and T. L. Lochs, Eds., Academic Press, San Diego, CA, 1997, chap. 8.

[3] K. J. Williams and R. D. Esman, Electron. Lett., 30, 1965 (1994).

[4] S. Norcia, S. Tonda-Goldstein, R. Frey, D. Dolfi, and J. P. Huignard, Opt. Lett., 28, 1888 (2003). 
[5] D. Mongardien, C. Moronvalle, F. Deborgies, IEEE MTT S International Microwave Symposium Digest 2, 731-4 (1993).

[6] P. Goldgeier and G. Eisenstein, IEEE Microwave and Guided Wave Letters 9, 40, (1999)

[7] G. P. agrawal, Nonlinear fiber optics, Academic Press, N. Y., 1995, chap. 8.

[8] B. Zhu, L. Leng, L. E. Nelson, Y. Quian, L. Cowsar, S. Stulz, C. Doerr, L. Stuz, S. Chandrasekhar et al., Electron. Lett., 37, 844 (2001).

[9] Y.R. Shen, The principles of nonlinear optics, John Wiley \& Sons, N.Y. 1984., chap.10.

[10] G.P. Agrawal, Fiber optic communication systems, Wiley-Interscience, N.Y. 2002, chap.6.

[11] K. Tai, A. Hasegawa, A. Tomita, Phys. Rev. Lett. 56135 (1986)

[12] C.R.S. Fludger, V. Handerek, R.J. Mears, IEEE Journal of Lightwave Technology 19, $1140(2001)$

[13] R.G Smith, Applied Optics, 11, 2489 (1972). 